\title{
Malignant Parapharyngeal Neoplasm
}

National Cancer Institute

\section{Source}

National Cancer Institute. Malignant Parapharyngeal Neoplasm. NCI Thesaurus. Code C162823.

A primary or metastatic malignant neoplasm that affects the parapharyngeal space. 